\title{
Mitteilungen des GRC und ARC
}

\section{Deutscher \\ Rat für \\ Wiederbelebung}

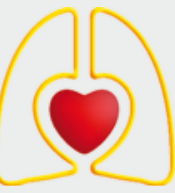

German

Resuscitation

Council

Deutscher Rat für Wiederbelebung -

German Resuscitation Council (GRC) e.V.

c/o Sektion Notfallmedizin, Universitätsklinikum Ulm

Pritzwitzstraße 43, 89070 Ulm

geschaeftsstelle@grc-org.de

www.grc-org.de

\section{Redaktion}

Dr. rer. nat. Dr. med. B. Dirks

Dr. med. S. Herdtle (sh)

Notfall Rettungsmed 2013 · 16: 492-492

DOI 10.1007/s10049-013-1778-7

๑) Springer-Verlag Berlin Heidelberg 2013

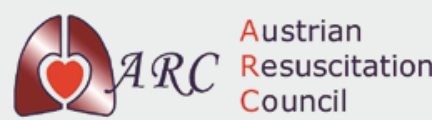

Österreichischer Rat für Wiederbelebung

Austrian Resuscitation Council -

Österreichischer Rat für Wiederbelebung (ARC)

p.A. conventa Veranstaltungsmanagement

Villefortgasse 22, A-8010 Graz

office@arc.or.at

www.arc.or.at

Redaktion

Univ. Doz. Dr. med. M. Baubin

Dr. med. J. Schlieber

\section{Öffentliche ERC Kurse in Österreich, geplant für 2014}

\section{Aus dem Course Management System des ERC und dem ARC Kurskalender}

\section{Advanced Life Support (ALS) Provider Kurse}

\begin{tabular}{|l|l|l|}
\hline 21.02.2014-23.02.2014 & $\begin{array}{l}\text { Ausbildungszentrum } \\
\text { Cap Wörth }\end{array}$ & $\begin{array}{l}\text { Velden am } \\
\text { Wörther See }\end{array}$ \\
\hline 28.04.2014-30.04.2014 & Bildungsinstitut Grillhof & Innsbruck/Vill \\
\hline 01.05.2014-03.05.2014 & $\begin{array}{l}\text { Bildungs-Center } \\
\text { des Roten Kreuzes }\end{array}$ & Feldkirch \\
\hline 04.07.2014-06.07.2014 & Rotes Kreuz Kramsach & Kramsach \\
\hline 26.09.2014-28.09.2014 & $\begin{array}{l}\text { Ausbildungszentrum } \\
\text { Cap Wörth }\end{array}$ & $\begin{array}{l}\text { Velden am } \\
\text { Wörther See }\end{array}$ \\
\hline 03.10.2014-05.10.2014 & $\begin{array}{l}\text { Ausbildungszentrum } \\
\text { der Johanniter }\end{array}$ & Wien \\
\hline 14.11.2014-16.11.2014 & Bildungsinstitut Grillhof & Innsbruck/Vill \\
\hline
\end{tabular}

European Pediatric Life Support (EPLS) Provider Kurse

\begin{tabular}{|l|l|l|}
\hline 21.02.2014-23.02.2014 & $\begin{array}{l}\text { Ausbildungszentrum } \\
\text { der Johanniter }\end{array}$ & Wien \\
\hline 08.05.2014-10.05.2014 & $\begin{array}{l}\text { Bildungszentrum } \\
\text { St. Magdalena }\end{array}$ & Linz \\
\hline 13.06.2014-15.06.2014 & $\begin{array}{l}\text { Bildungs-Center } \\
\text { des Roten Kreuzes }\end{array}$ & Feldkirch \\
\hline 05.09.2014-07.09.2014 & $\begin{array}{l}\text { Ausbildungszentrum der } \\
\text { Johanniter }\end{array}$ & Wien \\
\hline 24.10.2014-26.10.2014 & $\begin{array}{l}\text { Ausbildungszentrum } \\
\text { Schloss St. Georgen }\end{array}$ & $\begin{array}{l}\text { St. Georgen } \\
\text { am Längsee }\end{array}$ \\
\hline 21.11.2014-23.11.2014 & $\begin{array}{l}\text { Ausbildungszentrum } \\
\text { der Johanniter }\end{array}$ & Wien \\
\hline & &
\end{tabular}

Neonatal Life Support (NLS) Provider Kurse

\begin{tabular}{|l|l|l|}
\hline 08.03.2014 & $\begin{array}{l}\text { Ausbildungszentrum } \\
\text { der Johanniter }\end{array}$ & Wien \\
\hline 13.09 .2014 & $\begin{array}{l}\text { Ausbildungszentrum } \\
\text { der Johanniter }\end{array}$ & Wien \\
\hline 11.10 .2014 & Kinderzentrum Wels & Wels \\
\hline 08.11 .2014 & $\begin{array}{l}\text { Ausbildungszentrum } \\
\text { Schloss St. Georgen }\end{array}$ & $\begin{array}{l}\text { St. Georgen } \\
\text { am Längsee }\end{array}$ \\
\hline 13.12 .2014 & $\begin{array}{l}\text { Ausbildungszentrum } \\
\text { der Johanniter }\end{array}$ & Wien \\
\hline
\end{tabular}

Generic Instructor Kurse (GIC)

\begin{tabular}{|l|l|l|}
\hline 28.03.2014-29.03.2014 & $\begin{array}{l}\text { Seminarhotel Springer- } \\
\text { Schlößl }\end{array}$ & Wien \\
\hline 12.12.2014-13.12.2014 & Bildungsinstitut Grillhof & Innsbruck/Vill \\
\hline
\end{tabular}

